\title{
Experiencias institucionales y agencias de jóvenes de sectores populares en Buenos Aires
}

\section{Institutional Experiences and Agencies of Young People from Popular Sectors in Buenos Aires, Argentina}

Pablo Francisco Di Leo

\begin{abstract}
Resumen
Este artículo presenta resultados de una investigación social financiada cuyo objetivo principal es analizar las experiencias y agencias de personas jóvenes de sectores populares en instituciones que propician el acceso y ejercicio de derechos en el Área Metropolitana de Buenos Aires (AMBA), Argentina. Para la construcción de datos se desarrolló una estrategia metodológica cualitativa, mediante entrevistas en profundidad y narrativas biográficas con catorce jóvenes en cinco instituciones. En este trabajo se presentan tres categorías emergentes del análisis de los relatos juveniles, denominadas narrativas del yo: descubrimiento, (re)orientación moral, transformación colectiva. En torno a estas narrativas se aborda en términos teóricos el problema de las agencias y las construcciones identitarias juveniles, utilizando herramientas conceptuales de las sociologías de la experiencia (Dubet 2013), del individuo (Araujo y Martuccelli 2012) y la teoría social contemporánea (Ema López 2004; Mahmood 2006; Taylor 2006; Butler 2012; Heller 2017). Como concluye el artículo, estas instituciones suministran a las y los jóvenes diversos soportes que les abren oportunidades para resignificar y ejercen sus derechos en territorios socialmente vulnerabilizados. Construyen así formas de agencia y de ejercicio de la ciudadanía que no responden a definiciones teóricas o legales abstractas, sino que se anclan en sus experiencias, maneras cotidianas de convivir y proyectar con otras personas.

Palabras clave: Agencia juvenil, Identidad narrativa, Jóvenes, Sectores populares, Experiencias institucionales.
\end{abstract}

\begin{abstract}
This article presents results of a funded social research which main objective is to analyze the experiences and agencies of young people from popular sectors in institutions that promote the access and the exercise of rights in the Metropolitan Area of Buenos Aires (MABA), Argentina. For the data construction the researchers conducted, as a qualitative methodological strategy, in-depth interviews and biographical narratives with fourteen young people in five institutions. This work presents three categories emerging from the analysis of youth narrations, called self-narratives: discovery, moral (re) orientation, and collective transformation. Around these narratives is discussed in theoretical terms the problem of young people agencies and identity constructions, using conceptual tools from the sociologies
\end{abstract}

1. Consejo Nacional de Investigaciones Científicas y Técnicas (CONICET); Instituto de Investigaciones Gino Germani; Facultad de Ciencias Sociales, Universidad de Buenos Aires (UBA), Argentina,pfdileo@gmail.com

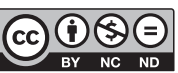


of experience (Dubet 2013), the individual (Araujo y Martuccelli 2012), and the contemporary social theory (Ema López 2004; Mahmood 2006; Taylor 2006; Butler 2012; Heller 2017). The article concludes that these institutions provide young people with various supports that open up opportunities to re-signify and exercise their rights in socially vulnerable territories. In this way, they build agencies and exercise their citizenship in ways that do not respond to abstract theoretical or legal definitions, but are anchored in their experiences, everyday ways of cohabiting and projecting along with other people. KeyWord: Youth agency, Narrative identity, Young people, Popular sectors, Institutional experiences.

\section{Introducción}

A partir de las transformaciones sociales vividas desde fines del siglo XX en nuestra región, se multiplicaron las desigualdades socioeconómicas, territoriales, étnicas, de género y raza que atraviesan las experiencias vitales juveniles. Desde la profunda crisis socioeconómica del 2001 hasta la actualidad, en el Área Metropolitana de Buenos Aires (AMBA) ${ }^{1}$, Argentina, los sectores populares ${ }^{2}$ se configuran mediante diversas dinámicas económicas, políticas y territoriales que generan heterogeneidades entre individuos y grupos, a la vez que multiplican vulnerabilidades, carencias y demandas que se acumulan (Cravino 2008). Las posibilidades de acceso y ejercicio a los derechos de educación, la salud y el trabajo regulado develan múltiples desigualdades sociales e institucionales (Chaves, Fuentes y Vecino 2016; Piovani y Salvia 2018). En estas condiciones, las y los jóvenes enfrentan en sus vidas distintas pruebas sociales e inconsistencias posicionales, frente a las cuales despliegan sus agencias y, en ese proceso, se van produciendo como individuos y ciudadanos (Araujo y Martuccelli 2012; Di Leo y Camarotti 2017).

Desde hace varias décadas, las ciencias sociales en América Latina abordan la condición juvenil a partir de la noción de generación, la cual alude a aquellos aspectos socio-históricos que delimitan marcos comunes de socialización; así como la capacidad de las personas para la comprensión e identificación de una serie de desafíos comunes que se les presentan en un momento histórico dado (Vommaro 2015). Desde esta perspectiva, diversos estudios desarrollados en Argentina analizan las características y las heterogeneidades presentes en las experiencias institucionales juveniles. Si bien cada vez más jóvenes de sectores populares transitan diversas instituciones públicas (especialmente escolares), en escasas ocasiones pueden desplegar en ellas sus agencias y procesos de individuación (Paulín y Tomasini 2014; Núñez y Litichever 2015; Gentile 2017; di Napoli 2018; Said 2018).

En este artículo presento resultados de un proyecto de investigación financiado ${ }^{3}$ cuyo objetivo principal es analizar las experiencias y agencias en jóvenes de sectores populares del AMBA en instituciones que propician el acceso y ejercicio de sus derechos. En la siguiente sección reseño algunas herramientas conceptuales que utilizo para el despliegue del problema de investigación. Luego presento la estrategia metodológica seguida para la construcción y análisis de los datos. Seguidamente desarrollo los resultados del estudio, centrándome en tres categorías emergentes del análisis de los relatos juveniles, a las que denomino narrativas del yo: descubrimiento, (re)orientación moral, transformación colectiva. En torno a estas narrativas abordo en términos teóricos el problema de las agencias y las construcciones identitarias de personas jóvenes, utilizando conceptos de las sociologías de la experiencia (Dubet 2013), del individuo (Araujo y Martuccelli 2012) y la teoría social contemporánea (Ema López 2004; Mahmood 2006; Taylor 2006; Butler 2012). En las conclusiones sintetizo y articulo los resultados, reflexionando sobre las tensiones y potencialidades presentes en estas experiencias institucionales y sus implicancias en las agencias, en las identidades y en las formas de ejercicio de derechos de jóvenes en territorios socialmente vulnerabilizados.

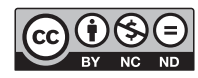




\section{Herramientas conceptuales}

Según François Dubet (2013), el concepto de institución designa la mayor parte de los hechos sociales que están organizados mediante símbolos, rituales, valores y normas, que se transmiten de una generación a otra y se imponen a los individuos. Son todas las actividades regidas por anticipaciones estables y recíprocas. No son únicamente hechos y prácticas colectivas, sino también marcos cognitivos y morales dentro de los cuales se desarrollan los pensamientos individuales. Las actuales transformaciones producidas en gran parte de las sociedades modernas - con intensidades diversas- no provocan solo una crisis de las instituciones encargadas de la socialización, una prueba de su capacidad de adaptación a nuevas situaciones, sino que generan un movimiento profundo de desinstitucionalización: una nueva modalidad de vinculación entre valores, normas e individuos; es decir, una nueva forma de socialización. Los valores y las normas ya no pueden ser representados como entidades trascendentales, ahistóricas, por encima de los individuos. Aparecen como producciones sociales, conjuntos compuestos por principios de integración múltiples y a menudo contradictorios.

Por ende, el proceso de desinstitucionalización genera la separación de dos procesos que la sociología clásica confundía: la socialización y la subjetivación. Las instituciones socializadoras estudiadas por las ciencias sociales -como la familia y la escuela entre otras- se han vuelto problemáticas, inmanentes, (re)creadas y (re)significadas por las personas en sus experiencias y relaciones cotidianas. Dichas instituciones ya no pueden asegurar la transmisión intergeneracional de sus símbolos, rituales, valores y normas de manera estable y homogénea, sino que permanentemente son cuestionadas, desestabilizadas y resignificadas a partir de las experiencias singulares de las nuevas generaciones.

Como lo social y lo institucional ya no tienen unidad, coherencia o sentidos a priori, Dubet (2013) propone colocar en el centro de los análisis sociológicos al concepto de experiencia, que define como la manera que tienen los individuos de construir lo social y de construirse a sí mismos. Las personas deben, de manera más o menos consciente y rutinaria, resolver problemas y dominar su posición en la sociedad. Las experiencias sociales e institucionales no son ni totalmente determinadas ni totalmente libres.

Construimos nuestras experiencias sociales articulando tres heterogéneas lógicas de la acción: a) integración: en la construcción de nuestras experiencias individuales sintetizamos un conjunto de roles y de pertenencias colectivas, el género, la profesión, la familia, las creencias, la raza, las culturas; b) estrategia: nuestras identidades son también un conjunto de recursos y capitales que movilizamos en intercambios sociales particulares; c) subjetivación: nos definimos también como individuos porque las sociedades modernas recrean y proponen representaciones culturales en torno a la categoría de sujeto, en tanto ser genérico que detenta atributos de creatividad, autonomía, libertad y que, paradójicamente, se distancia de la sociedad.

En este sentido, Charles Taylor (2006) parte de una crítica de los preconceptos científicos naturalistas que buscan analizar la construcción de la identidad del yo de manera «objetiva», independientemente de las descripciones o interpretaciones que hagan las personas sobre sí mismas y sin referencias a sus entornos. En cambio, plantea que «solamente somos yos en esas cuestiones concretas que son importantes para nosotros. Lo que soy como un yo, mi identidad, está esencialmente definido por la manera en que las cosas son significativas para mí. Y, como ya se ha analizado ampliamente, esas cosas son significativas para mí y el asunto de mi identidad se elabora, solo mediante un lenguaje de interpretación que he aceptado como válida articulación de estas cuestiones» (Taylor 2006, 61).

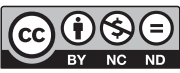


Esto lleva a otro aspecto central de la identidad personal: únicamente se hace posible en una comunidad lingüística, en interacción con otras personas. La respuesta a la pregunta «¿quién soy yo?», adquiere sentido en una comunidad de hablantes. Defino mi identidad personal en relación con interlocutores, compañeras y compañeros de conversación que fueron o son esenciales para la construcción de mi propia autodefinición. El yo se conforma dentro de una urdimbre de interlocución, en un diálogo permanente con las visiones y las acciones de las y los otros. Aquí Taylor (2006) introduce otra dimensión constitutiva de las identidades: los marcos referenciales. Ante la pregunta, «¿quién soy yo?», respondo mencionando aquello que es importante para mí, lo que da sentido a mi vida. Mi identidad se va construyendo en relación a los compromisos, las identificaciones que me proporcionan el lenguaje, el marco y el horizonte desde los cuales establezco, caso a caso, lo que es bueno, valioso, lo que vale la pena vivir, lo que hay que hacer, lo que defiendo o a lo que me opongo. Los marcos referenciales son los supuestos de fondo y los contextos en relación a los cuales el yo puede tomar una postura, identificarse, actuar y formular juicios morales.

Los diversos marcos referenciales y orientaciones del yo no necesariamente se excluyen entre sí y suelen combinarse a lo largo de su vida. Es importante para el individuo que se articulen con la comprensión que tiene de su vida como una historia, una narrativa, que se despliega en contextos institucionales, colectivos, políticos; en espacios privados y públicos, atravesados por conflictos, disputas y negociaciones con otras personas, «es decir, que lo pertinente es considerar a los discursos que expresan las identidades (y que están en el origen de muchas acciones y/o disposiciones para la acción) como la resultante siempre provisoria de la presencia de los demás en nosotros y de los intentos de nosotros por estar presentes en nuestros propios términos en los demás y en los múltiples registros de lo social; impulsos de una doble presencia que revela la problemática de la autonomía y la heteronomía en la construcción de la identidad» (Meccia 2016, 61).

Paul Ricoeur (1996) define el concepto de identidad narrativa a partir de una dialéctica entre dos dimensiones (solo diferenciables analíticamente): la identidad-idem, que constituye la seguridad ontológica, la continuidad existencial del individuo; y la identidad-ipse, proceso de identificación nunca cerrado, en el que ocupan un lugar fundamental las relaciones cotidianas del sí mismo con las demás personas.

Para el abordaje sociológico de las complejidades y vinculaciones entre las experiencias institucionales, los marcos referenciales y las identidades juveniles también recurrimos a los aportes analíticos de Danilo Martuccelli (2007) en el campo de la sociología de la individuación: una sociología a escala de los individuos, que tiene como horizonte el estudio de las capacidades existenciales y sociales de las personas de sostenerse en el mundo (Araujo y Martuccelli 2012). En sus estudios empíricos desarrollados en Francia y Chile utiliza como herramienta el concepto de soportes, entendidos como «(...) aquellos conjuntos heterogéneos de elementos, reales o imaginarios, que se despliegan a través de un entramado de vínculos, que suponen un diferencial de implicación según las situaciones y las prácticas, y gracias a los cuales los individuos se sostienen» (Martuccelli 2007, 81-82). Todas las personas construimos nuestras ecologías existenciales (Martuccelli 2007) utilizando y combinando diversos tipos de soportes afectivos, materiales y simbólicos, desplegados en su experiencia biográfica en un entramado elástico de vínculos sociales e institucionales.

Dialogando con estas herramientas conceptuales, nuestra investigación busca aportar a la visibilización de las complejidades presentes en los procesos de individuación de jóvenes de sectores

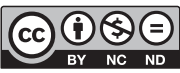


populares del AMBA, a partir del análisis de sus experiencias en instituciones que propician el acceso y ejercicio de derechos y en las que eligen participar. En este artículo me centro en los siguientes problemas: ¿cómo construyen las personas jóvenes de sectores populares sus experiencias e identidades en relación a dichas instituciones?; ¿qué narrativas del yo, marcos referenciales y soportes despliegan en torno a dichas experiencias?; ¿qué características y tensiones entre autonomías y heteronomías presentan sus agencias?

\section{Estrategia metodológica}

Para la construcción y análisis de los datos se desarrolló una estrategia metodológica de tipo cualitativo, ya que es la que mejor se articula con el paradigma interpretativo (Vasilachis de Gialdino 2007), permitiendo aproximarse a los procesos de construcción de las experiencias y agencias juveniles. Se utilizó como técnica principal la entrevista en profundidad, pues mediante la misma es posible construir datos en torno a las experiencias individuales y grupales, indagando sobre prácticas, significaciones y reflexiones en torno a sí mismas, las y los otros y los espacios sociales e institucionales en los cuales desarrollan sus interacciones cotidianas. Se buscó que las personas entrevistadas reflexionen en torno a sus propias experiencias personales e institucionales, procurando captar polifonías, tensiones, temáticas emergentes y articulaciones intra e inter-discursivas (Giarracca y Bidaseca 2004).

Para la reconstrucción de las experiencias juveniles se retomó el enfoque biográfico, que consiste en el despliegue narrativo de las vivencias de los individuos a lo largo del tiempo con el objeto de elaborar, a través de entrevistas sucesivas, un relato que permita mostrar «(...) el testimonio subjetivo [buscando dar cuenta] tanto de los acontecimientos como de las valoraciones que dicha persona hace de su propia existencia» (Pujadas Muñoz 1992, 47-48). Retomando las propuestas de Michèle LeclercOlive (2009), en la primera entrevista se solicitó a las y los jóvenes que identificaran las vivencias que consideraran como más significativas a lo largo de sus experiencias institucionales. Los acontecimientos o giros biográficos permiten identificar los momentos de bifurcación o de cambios importantes en las experiencias individuales. Dichos sucesos son seleccionados, descritos y evaluados por las personas a la luz de sus experiencias posteriores. Por ende, desde esta perspectiva el relato biográfico detenta un carácter performativo: no recupera ni representa una historia, sino que la instituye desde el presente en virtud de la selección de los acontecimientos significativos por parte de las y los entrevistados (Di Leo y Camarotti, 2017).

Se incluyeron en la muestra a varios contextos barriales, instituciones y sujetos para realizar un análisis comparativo de las experiencias personales e institucionales, sin perder de vista las condiciones socioeconómicas y urbanas en las que viven (Vasilachis de Gialdino 2007). En función de la estrategia del muestreo teórico de la teoría fundamentada (grounded theory), la determinación de la muestra final se articuló con la identificación y despliegue de las categorías centrales emergentes, sus propiedades y articulaciones, en un ida y vuelta constante entre los datos y la teoría (Strauss, Corbin y Zimmerman 2002). La muestra quedó constituida por catorce jóvenes, ocho mujeres y seis varones, que viven en diversos barrios populares del AMBA y que participan - en calidad de estudiantes o cursantes- en diversas actividades educativas, recreativas, deportivas, culturales o laborales en cinco instituciones que propician el acceso y ejercicio de derechos. ${ }^{4}$ En la Tabla 1 se indica la conformación de la muestra de jóvenes e instituciones:

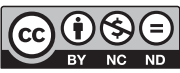




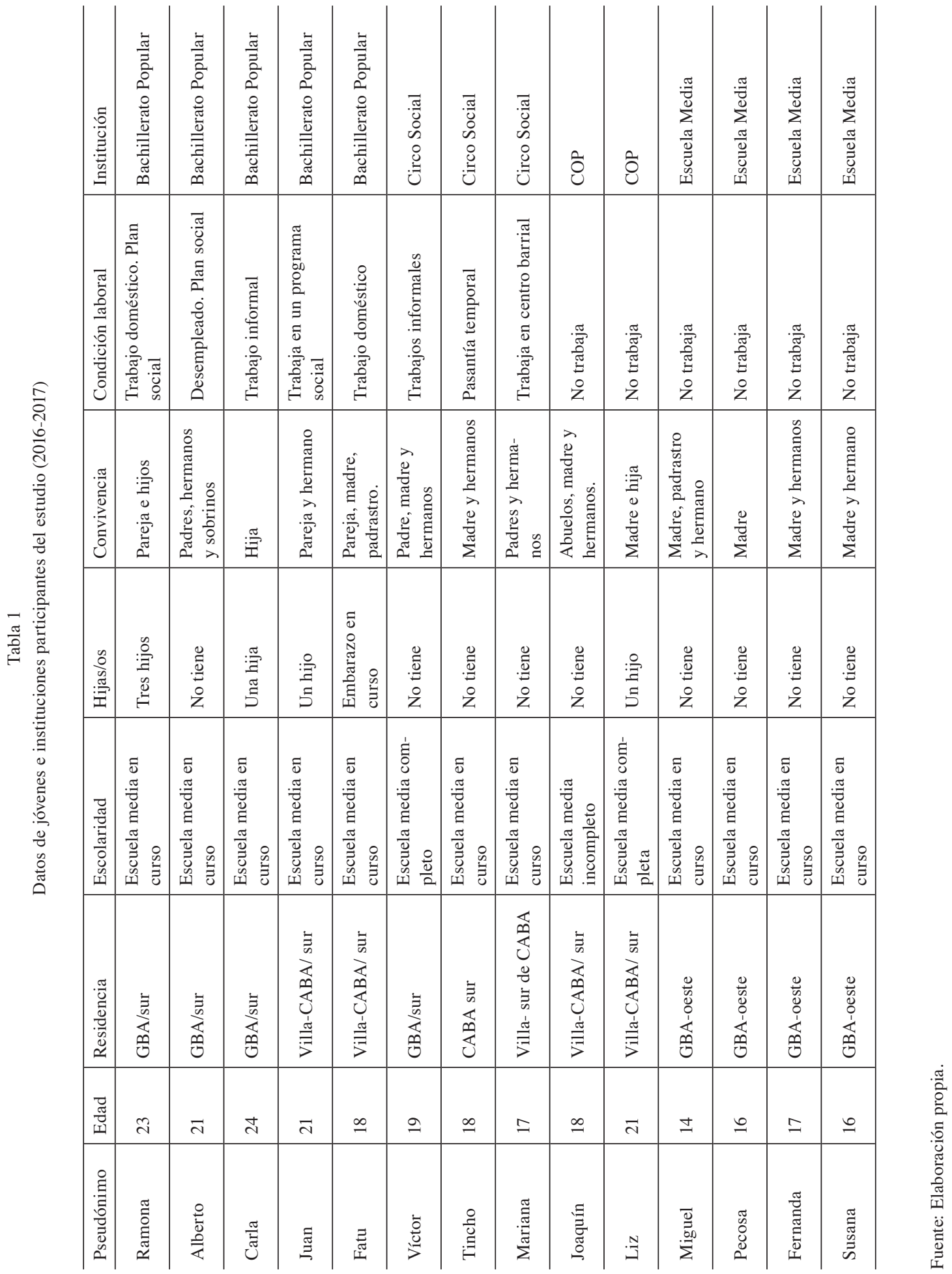


Como se indica en la Tabla 1, el trabajo de campo se desarrolló durante 2016 y 2017 en las siguientes instituciones:

- Dos bachilleratos populares - escuelas medias para jóvenes y adultos que se definen a sí mismas como autogestionadas y populares, con autonomía política y pedagógica respecto del Estado (Said 2018)-: uno ubicado en un barrio marginalizado de la Ciudad Autónoma de Buenos Aires (CABA) y otro en el Gran Buenos Aires (GBA).

- Circo Social: organización que propicia el acceso a actividades culturales y deportivas, ubicada en la CABA.

- COP: institución dependiente del Gobierno de la CABA, dedicada a la capacitación laboral.

- Una escuela media que incluye en su proyecto institucional la educación no formal, la formación profesional y la integración de jóvenes en contextos de vulnerabilidad social en el GBA.

Como se observa en la Tabla 1, para la selección de las personas jóvenes a incluir en el estudio, se buscó una heterogeneidad con respecto a: sus inserciones territoriales, edades, género, escolaridad, condición familiar y laboral. Sin embargo, la muestra final no se determinó a partir de cuotas según dichas variables, si no que se terminó de conformar a partir de la voluntad de los sujetos de participar (tanto a nivel individual como institucional) en la investigación y -como se indica arriba- del criterio de saturación teórica, que consideramos el más adecuado para una investigación social cualitativa (Strauss, Corbin y Zimmerman 2002). ${ }^{5}$

Siguiendo los lineamientos generales de la teoría fundamentada, a partir de la estrategia de la comparación constante, se construyeron y codificaron los datos de manera simultánea, utilizando como auxiliar el software de análisis textual Atlas ti (Vasilachis de Gialdino 2007). Al finalizar el trabajo de lectura y codificación del corpus de entrevistas, ya se habían identificado varias categorías emergentes. Para el despliegue y vinculación de dichas categorías, se utilizaron herramientas conceptuales de la teoría social, así como datos provenientes de otras investigaciones de nuestro equipo o de otros estudios desarrollados en la región. Se aplicaron los criterios de parsimonia -buscando maximizar la comprensión de un fenómeno con el mínimo de conceptos posible- y de alcance, procurando ampliar el campo de aplicación del análisis sin desligarse de la base empírica (Strauss, Corbin y Zimmerman 2002). En la siguiente sección presento resultados del análisis de las entrevistas a jóvenes, organizados en torno a las tres categorías emergentes a las que denomino narrativas del yo: descubrimiento, (re)orientación moral, transformación colectiva.

\section{Resultados}

\section{Narrativas de descubrimiento}

En los relatos en torno a sus experiencias institucionales, las y los jóvenes destacan, en primer lugar, su sorpresa al descubrir lugares totalmente distintos a aquellos que frecuentan en su vida cotidiana; y que desconocían de su existencia en sus barrios. Para Fatu ${ }^{6}$ y Mariana, las instituciones en las que participan se contraponen con dos ámbitos que conocen especialmente: la calle y la escuela.

Fatu (F): Para mí es más seguro acá [en el bachillerato popular] porque no es lo mismo. Porque allá [en la escuela pública a la que asistía] todo lo que a mí me enseñaban y, sobre todo, la calle... no me gustaba.

Entrevistadora: ¿Por qué?

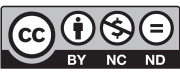


F: Porque era muy peligroso... Como yo estaba en turno noche salía muy tarde y te robaban ahí...

Porque últimamente el barrio está como que, más chiquitos, más se drogan, más andan en cualquier cosa, ¿entendés? $\mathrm{Y}$ últimamente hay mucha droga y hay mucho quilombo. Entonces muchas veces es preferible evitar esas cosas y esas relaciones y tener una relación sana, por así decirlo, con gente de afuera (Mariana).

Jóvenes como Fatu o Mariana nacieron en barrios marginalizados del AMBA -también denominados villas $^{7}-$ a finales del siglo XX e inicios del nuevo milenio. En dicho período, se manifestaron en Argentina las consecuencias más extremas de los procesos de debilitamiento o desmantelamiento de las instituciones estatales y laborales que, en décadas anteriores, disminuían las inseguridades individuales y sociales. En estos barrios construyen casi la totalidad de sus experiencias, y se edifican material y simbólicamente, a partir de una multiplicidad de prácticas, relaciones, conflictos y formas de solidaridad que generan diversos vínculos y fronteras a partir de valoraciones, marcas identitarias, sociales y afectivas (Chaves, Fuentes y Vecino 2016; Di Leo y Camarotti 2017).

Si bien la calle se configura como un mundo social complejo, asociado a experiencias y acontecimientos biográficos contrapuestos, es de destacar que las y los jóvenes de sectores populares entrevistadas vinculan este espacio a la inseguridad, las violencias y los consumos problemáticos de drogas (Gentile 2017). Las personas habitantes en barrios marginalizados del AMBA son víctimas de los mayores índices de delitos violentos de Argentina. Simultáneamente, sufren la escasez, la ausencia o la desconfianza de las instituciones estatales -especialmente la policía-, por lo cual las redes de sociabilidad barrial -basadas principalmente en vínculos familiares- constituyen casi sus únicos soportes sociales. En estas condiciones, los conflictos, crisis o rupturas en dichas redes profundizan los sentimientos de vulnerabilidad e inestabilidad subjetiva e incertidumbre hacia el futuro en sus habitantes. Un tercer fenómeno articulado con los dos anteriores, recae en la reiteración de los delitos en estos territorios, impactando fuertemente en las redes de sociabilidad barrial, profundizando y encadenando conflictos o desconfianzas entre vecindades, amistades o familiares, lo que magnifica aún más los sentimientos de inseguridad de sus habitantes (Auyero y Berti 2013; Kessler y Bruno 2018).

Frente a estas condiciones de violencias y vulnerabilidades encadenadas, las y los jóvenes despliegan diversas agencias en sus formas de habitar, vincularse o circular en estos territorios. Especialmente las mujeres, como Mariana, elaboran complejas estrategias para entrar, salir o circular por el barrio, dirigidas a evitar o disminuir distintos riesgos:

Entrevistadora: ¿Y vos dentro del barrio tenés lugares por dónde vas o por dónde no? ¿Hay una forma como de moverse?

Mariana: Para entrar a mi casa hay dos entradas. La de parte de una canchita y la entrada general. Y por lo general si es de noche o si salgo sola entro por la entrada general que es donde se moviliza más gente. Y si salgo con alguien, salgo por el otro lado, que me queda más cerca de la parada [del colectivo] pero, por lo general, no ando sola por ahí porque es donde se juntan más los chicos que fuman. Entonces, para evitar ciertas cosas es preferible no estar sola.

Por otro lado, sus experiencias escolares se asocian a distintas formas de estereotipos, discriminación y negación del reconocimiento por sus orígenes étnicos, nacionales, barriales o por el grupo social de pertenencia. Las personas jóvenes narran situaciones de «repetición de años», «fracasos», segregaciones o exclusiones escolares. Como surge de diversos estudios realizados durante los últimos años en Argentina, la relación entre jóvenes de sectores populares y escuela media se encuentra atravesada por múltiples fronteras

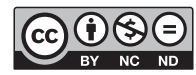

Rev. Reflexiones 98 (2) Julio-Diciembre, 2019: 141-158, E-ISSN: 1659-2859 
simbólicas y materiales que generan trayectorias escolares de baja intensidad, su exclusión o su inclusión en fragmentos devaluados del sistema educativo (Núñez y Litichever 2015; Chaves, Fuentes y Vecino 2016). Se reproducen así, a través de diversas mediaciones institucionales, las desigualdades sociales:

\begin{abstract}
Además, en la otra escuela no me gustaba como enseñaban. Porque allá te dicen, te explican y si no entendés, bueno, jodete. No les importa, y acá [en el bachillerato popular] sí. Acá te lo vuelven a explicar 50 veces si querés, hasta que vos lo entiendas. Y cuando un compañero lo entiende, los compañeros te explican si no lo entendés. Y si no, lo hacemos todos juntos hasta que vos entiendas. En las escuelas normales no es así: ellos te explican una o dos veces y ya está, si no entendiste, bueno, ya es tu problema. Y acá no es así y eso es lo que más a mí me gusta de acá. Si necesitás un consejo de ellas [las profesoras], ellas te dicen, te hablan y te aconsejan: «esto está mal», «está bien que estás haciendo esto», «está bien que estás mejorando»... Y se preocupan si vos estás enferma o no estás enferma. En los colegios otros normales, no son así. "Bueno, si no venís es porque vos no quisiste, tenés que traer esto, lo otro si estás enfermo", no se preocupan por vos. Y las docentes de acá sí se preocupan. Si le pasa algo a algún compañero que no está viniendo vamos todos a su casa a ver qué le está pasando (Fatu).
\end{abstract}

Como sintetizan Fatu, Mariana y Carla, las experiencias juveniles en las instituciones donde realizamos nuestro trabajo de campo se presentan como excepciones, refugios en los que eligen participar cotidianamente porque se sienten reconocidos «como personas y no como un número más», habilitando diversos soportes simbólicos, afectivos, materiales y repertorios de acción no violentos ni discriminatorios, que los ayudan a (re)vincularse con otras personas jóvenes y adultas y, en general, con organizaciones sociales e instituciones públicas:

Lo más importante [del circo social] es el espacio de contención que tiene, o sea, desde que vos llegás y básicamente sos como otra, sos vos misma. Podés expresarte, podés decir lo que te pasa, podés enojarte, llorar, estar feliz y nadie te va a juzgar por eso, o sea, te van a bancar o te van a dar una mano, o sea, es ese espacio de contención que tienen al darte un oído, al darte una mano cuando te pasa algo, más allá de todas las estructuras y todo lo que haya (Mariana).

Acá todo se charla y si hay algo que no te gusta te termina atrapando, te termina gustando, o le encuentran la manera como para que sientas interés o te explican en qué te puede ayudar. Entonces está bueno eso, es cotidiano. Todos los días ves algo distinto en el bachi [bachillerato popular], no sabés con qué te vas a encontrar (Carla).

En este tipo de espacios institucionales se abren otras posibilidades para el despliegue de los procesos de subjetivación e individuación juveniles, esto a partir de vínculos democráticos, no violentos, ni discriminatorios (Said 2018). A su vez, desde esta dialéctica entre el sí mismo y el otro, las y los jóvenes pueden resignificar y reconstruir sus experiencias. En estos escenarios construyen narrativamente sus identidades y marcos referenciales personales en interacción con otras personas.

\title{
Narrativas de (re)orientación moral
}

En los relatos de Carla, Mariana, Tincho, Juan y Bautista se presentan diversos espacios y tiempos institucionales de encuentro, convivencia, trabajo colectivo, en los que se sienten escuchados y tratados como personas («no como un número más»), funcionando como desencadenantes o motores en la formación de sus identidades personales. Estos escenarios propician la construcción de relaciones de «confianza» con personas adultas:

Creo que en un ámbito educativo como la escuela, tenés un profesor que te da una materia y que te evalúa tu inteligencia con esa materia, con lo que vos rendís, esos 40 minutos te evalúa y te dice «bueno sos un 7, vos son un 8». En cambio un profesor que tenés toda la tarde, que lo conocés hace años y tenés mucha más confianza, tenés mucho

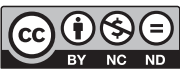

Rev. Reflexiones 98 (2) Julio-Diciembre, 2019: 141-158, E-ISSN: 1659-2859 
tiempo con esa persona y esa persona conoce lo que a vos te sale y conoce lo que te puede llegar a salir. Entonces no te dice: «Vos sos un roll» [un ejercicio circense], te dice: «vos podés hacer un roll». Acá podés ser la persona que realmente sos sin estar con la cabeza en otro lado, estás en el lugar (Mariana).

La confianza en sí mismas, en las instituciones y las otras personas constituye una dimensión fundamental en los procesos de construcción de nuestras identidades en la actual etapa de la modernidad. Nos permite construir nuestra seguridad ontológica, es decir, nuestro ser-en-el-mundo; sin el cual nos sería imposible actuar y habitar el mismo universo social con otras personas. Se desarrolla en conjunción con la formación de un sentido interno de confiabilidad, que provee posteriormente una base para nuestra auto-identidad estable (Giddens 1991). En los relatos juveniles, las relaciones de confianza se presentan como experiencias extraordinarias de apropiación subjetiva del sí mismas, las otras personas y del entorno institucional, que habilitan sentidos y orientaciones para sus prácticas o proyecciones:

\footnotetext{
Los pibes de acá [el bachillerato popular], yo siento que la ayuda los hace sentir a todos capaces. Porque todos somos capaces de poder hacer algo, tal vez hay pibes que vienen con la idea de terminar el secundario y listo. Pero se dan cuenta que sirven para muchas cosas y les interesa eso. Como que les hace clic la cabeza. «Yo puedo servir para esto» o «yo creo que soy bueno en esto» o «a mí me interesa esto». Yo creo que es fundamental cómo el bachi trabaja con los chicos, porque yo siento que es algo de ellos (Carla).
}

En estas experiencias emerge una concepción de la agencia y la autonomía del yo basadas en los vínculos intersubjetivos. Retomando la propuesta de Ema López (2004), podemos comprender la agencia a partir de la dialéctica entre lo individual y lo social: no es una mera propiedad personal, sino una posibilidad - poder hacer- compartida, habilitando a concepciones relacionales y abiertas del poder y las identidades. La acción emerge como mediación entre flujos de acciones. En fin, la agencia se manifiesta como potencialidad siempre abierta de (re)crear, transformar conexiones, relaciones entre entidades heterogéneas, rompiendo con el dualismo entre estructura y sujeto, individuo y sociedad. Dialogando con los trabajos de Saba Mahmood (2006), en estas narrativas se ponen de manifiesto concepciones de agencia y de autonomía que difiere de las definiciones de raigambre humanista-liberal: sinónimos de liberación o de resistencia frente a las relaciones de dominación que, según la orientación ideológica y el contexto histórico, se identifican con la religión, la tradición, el Estado, el mercado, el patriarcado, la familia o toda institución en general: «La agencia, desde este punto de vista, es entendida como la capacidad de cada persona para realizar sus intereses individuales, en oposición al peso de la costumbre, tradición, voluntad trascendental u otros obstáculos individuales y colectivos. Por lo tanto, el objetivo humanista de la autonomía y la expresión de las capacidades individuales constituye el sustrato, las cenizas durmientes que podrán encender en llamas bajo la forma de un acto de resistencia cuando las condiciones así lo permitan» (Mahmood 2006, 127, traducido por la persona autora). ${ }^{8}$

La identidad personal no existe como una esencia independiente, sino como un producto de las instituciones. Al mismo tiempo, hace posible la transformación esta última: el yo se conforma en una sociedad; pero también, de forma simultánea, el yo da materialidad e historicidad a la sociedad, participando en su permanente recreación. Por ende, la autonomía y la heteronomía no son estados puros ni excluyentes, sino que deben comprenderse como un continuo proceso, en permanente tensión, presente en toda construcción identitaria y en toda acción (Castoriadis 1997).

Al narrar sus experiencias institucionales, las y los jóvenes señalan diversos acontecimientos significativos que marcaron un antes y un después, un giro biográfico, en el que se modificaron sus sentidos y sus prácticas en torno a sí mismos, las otras personas y las instituciones. Fruto de su participación en el bachillerato popular, Carla pudo llevar adelante una separación con una pareja que limitaba sus posibilidades de autonomía, de agencia, de actuar de otra manera:

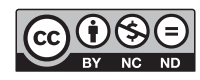

Rev. Reflexiones 98 (2) Julio-Diciembre, 2019: 141-158, E-ISSN: 1659-2859 
[En el bachillerato popular] me reencontraba conmigo misma y para mí eso fue buenísimo porque me di cuenta de un montón de cosas que sentía y no las podía comprender en un momento y las empecé a entender a medida que yo veía que tenía contención, que me explicaban, que había algo más que no sé, que lavar los platos y atender a tu hija en tu casa. Me hice mucho más independiente y me encontré a mí misma (Carla).

Este encuentro consigo misma es especialmente valorado por Carla y otras mujeres jóvenes que viven con sus parejas o familias de origen, ya que significa la posibilidad de salir del espacio doméstico y de las tareas de cuidado familiar en los que muchas veces las encierran los mandatos de género. En cambio, en el bachi Carla pudo reconstruir la estima de sí misma como mujer y redefinir sus proyectos y sueños, tendiendo a lo que para ella es la vida buena, que, según Ricoeur (1996, 184-85), «es, para cada uno, la nebulosa de ideales y de sueños de realización respecto a la cual una vida es considerada como más o menos realizada o como no realizada. Es el plano del tiempo perdido y del tiempo recobrado».

Como continúo profundizando en la próxima sección, las personas jóvenes van (re)definiendo lo que es importante para sí mismas, lo que vale la pena hacer y vivir, la vida buena (Ricoeur 1996); no de manera solipsista o aislada, sino a partir de diversas experiencias, acontecimientos significativos donde las otras personas y las instituciones ocupan un lugar central. Así van (re)construyendo su yo a partir de un arduo trabajo sobre sí. En estas narrativas no se presentan a las normas institucionales, valores morales, marcos referenciales como contrapuestos (o impuestos) a una voluntad individual, a un self natural o previo a las instituciones o los poderes. Por el contrario, dichos marcos que reorientan al yo hacia el bien o la felicidad, se presentan como condiciones de posibilidad para la emergencia y despliegue de sus agencias, para actuar de otra manera. A partir de la repetición cotidiana de diversas formas de elaboración del trabajo ético -estudiar, practicar ejercicios circenses, compartir con otros-, modalidades de acción dirigidas a sincronizar los comportamientos externos con las motivaciones internas, las y los jóvenes van constituyendo y ubicando al yo en una teleología del sujeto moral. Es decir, en marcos referenciales que dan coherencia y sentido a sus vidas.

\section{Narrativas de transformación colectiva}

En narrativas del yo como las de Carla, Fatu, Bautista, Mariana y Alberto se presenta a la ayuda, la escucha, el trabajo con y para otras personas como marcos referenciales altamente valorados, en relación a los cuales (re)definen sus orientaciones morales, sus proyecciones hacia la vida buena, lo que vale la pena hacer y vivir hacia el futuro:

Fatu (F): Igual, cuando termine acá [en el bachillerato popular] voy a seguir viniendo, no me voy a ir...

Entrevistadora: ¿Por qué decís así?

F: Porque me gusta, voy a venir a ayudar a los compañeros que están empezando este año, ayudar a los docentes. Teníamos un compañero que terminó el año pasado que vino a ayudar a la profesora de matemática. Como le gusta matemática, la ayudaba, y yo también puedo venir a ayudar a alguien, no sé... a cualquiera (Fatu).

Entonces está mucho eso del compromiso de ayudar a la otra persona de saber que la otra persona no puede hacer sus cosas si vos no estás, o vos no podés hacerlo si la otra persona no está. Entonces creo que eso es uno de los valores que más me quedó, de trabajar en equipo y de ser responsable de cada una de las cosas (Mariana).

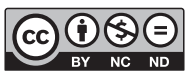

Rev. Reflexiones 98 (2) Julio-Diciembre, 2019: 141-158, E-ISSN: 1659-2859 
En estas narrativas de (re)orientación moral y ética, presentes tanto en mujeres y varones jóvenes, se articulan el cuidado del sí y el cuidado del otro, en un sentido cercano al analizado por Foucault (1996) en torno a la categoría de parresía (que retoma de la antigüedad griega clásica). Es así como la «parresía etimológicamente significa decirlo todo. La parresía lo dice todo; no obstante, no significa exactamente decirlo todo, sino más bien la franqueza, la libertad, la apertura que hacen que se diga lo que hay que decir, cómo se quiere decir y bajo la forma que se considera necesaria (...). Decir lo que se piensa, pensar lo que se dice, hacer que el lenguaje se corresponda con la conducta; esta promesa, esta especie de compromiso, están en la base de la parresía» (Foucault 1996, 88-89).

A partir de este compromiso, este acto de confianza, esta búsqueda de sintetizar lo que se dice, se piensa y se hace, surge un modo de subjetivación, una forma de relación del sujeto consigo mismo, que pone en el centro a la alteridad. La parresía permite hacer coincidir nuestros pensamientos con nuestras prácticas, pero solo en la medida en que es acción sobre nosotros junto con otros. Tal como se presenta en las narraciones juveniles, la construcción y transformación del yo solo se hace posible a partir de un simultáneo trabajo con y sobre las otras personas. El cuidado de sí es, al mismo tiempo, cuidado de otras y otros (Butler 2012). Los marcos referenciales que permiten la reconstrucción y reorientación moral del yo funcionan simultáneamente como orientadores éticos y soporte fundamental para el individuo.

Las y los jóvenes destacan en sus experiencias institucionales aquellos momentos y espacios en los que pueden debatir, reflexionar, planificar y concretar diversos proyectos para transformar colectivamente sus realidades:

Las actividades que más me gustan acá es cuando hacemos reuniones, la asamblea me gusta que tenemos los lunes. Más me gusta la asamblea porque podemos debatir todo, todo lo que está mal, lo que falta en el bachi y que tenemos que hacer en toda una semana, como las reuniones que hacemos acá, en el barrio, que siempre participa el bachi. Y me siento escuchada y me gusta porque la asamblea es donde tenés más información de todo lo que va a pasar en esta semana, aunque, ponele, vos no... Hay una reunión en el barrio y vos no sabés y en la asamblea te lo dicen, y es como que estás más informada de todo, de todo el barrio (Fatu).

Estas asambleas funcionan como espacios públicos que, según la conceptualización de Arendt (1993), son los ámbitos privilegiados para el ejercicio de la libertad de los sujetos -que requiere la visibilidad por y con otras personas-, ya que constituyen el territorio privilegiado de producción simultánea de intersubjetividades y singularidades. A partir de este diálogo e intercambio de experiencias, se abren nuevas posibilidades para articular los valores y las prácticas individuales con las normas y exigencias genérico-sociales. Como analiza Heller (2017), estos espacios intersubjetivos posibilitan la vinculación entre las dos perspectivas constitutivas de la identidad personal en las sociedades modernas: la inter$n a$-centrada en nuestra memoria autobiográfica- y la externa, basada en nuestros encuentros pasados con otras personas. Solo a partir del interjuego mediado institucionalmente entre estas dos perspectivas se hace posible la transformación del particular -encerrado en la pura reproducción inmediata de sus condiciones de vida, sin poder establecer una distancia crítica con respecto a ellas- en individuo. Este último, a partir del despliegue de los niveles de su ser ético, estético, filosófico y, fundamentalmente, político, tiene la posibilidad de reconocerse como ser genérico, estableciendo una relación creativa con su vida, con otras personas y con su entorno social e institucional.

El GBA cuenta con numerosos barrios populares, en los cuales hay carencias de infraestructura urbana y cuya población vive en situación de pobreza. En uno de esos barrios vive Alberto, quien tiene 21 años. Vive con su madre, su padre, dos hermanos (tiene otros dos más grandes que viven con sus propias familias) y dos sobrinos. Su madre es pensionada, su padre trabaja haciendo changas (empleo informal) y, aunque Alberto cobra un exiguo subsidio estatal para continuar con su escolaridad secundaria, también

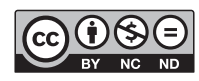

Rev. Reflexiones 98 (2) Julio-Diciembre, 2019: 141-158, E-ISSN: 1659-2859 
trabaja como mozo de viernes a domingo para ayudar en la economía familiar. En 2016 -año en que le hicimos la entrevista-, retomó sus estudios (los había interrumpido hace algunos años atrás, porque se le complicaba compatibilizar los horarios escolares y laborales) en un bachillerato popular cercano a su casa, en el que le permiten cursar de lunes a jueves, para poder ir a trabajar los viernes. Esta institución constituye para él -además de una posibilidad de ejercer su derecho a la educación secundaria- un verdadero refugio subjetivo y un valorado espacio de sociabilidad con otras personas jóvenes y adultas. Alberto sintetiza en el posible título de un libro colectivo, Orgullo de rubí, la fortaleza del nosotros, de la identidad colectiva construida con referentes y otras personas jóvenes en el bachillerato popular. Este trabajo colectivo que denota orgullo, permite sostener a la institución, pese a haber sufrido un incendio que destruyó por completo sus instalaciones:

Alberto (A): Ahora estamos haciendo como un libro, con la profesora de Lengua. Y estamos todos, todos, desesperados para hacer el libro...

Entrevistadora: ¿Qué vas a escribir vos?

A: Quiero proponer sobre la igualdad, contando cómo yo llegué al bachi, cómo me enteré por el amigo de mi hermano. Y que estuve sin estudiar, estuve trabajando hasta que él me dijo para anotarme. Le dije, «bueno, dale». digo, «sí, voy a estudiar» le dije. Al libro le pusimos en la tapa: «Orgullo de rubí». Porque es fuerte, indestructible. El rubí representa a los profesores que están acá y orgullo es nosotros por seguir y estar estudiando. Entonces el rubí representa...por más que se prenda fuego el colegio, nosotros seguiremos siendo duros, estamos duros como el rubí y eso nos une. Empezamos también así a dibujarle una mano para que sea una mujer que agarra un rubí, para tener igualdad (Alberto).

En estas experiencias institucionales juveniles, se despliegan las agencias y el poder a partir de la voluntad de los sujetos de querer vivir, trabajar y actuar orgullosamente en un espacio público, un escenario de reconocimiento intersubjetivo entre personas jóvenes y adultas. Se genera así el poder de reinventar un nosotros. Este Orgullo de rubí funciona como un tercero, un mediador colectivo en los vínculos intersubjetivos. A partir de estas experiencias institucionales, las y los jóvenes crean y se apropian de nuevos sentidos de la igualdad y la libertad. De esta manera, la ciudadanía deja de ser un concepto abstracto para constituirse en una manera concreta y cotidiana de convivir con otras personas (Dubet 2017).

\section{Conclusiones}

A partir del análisis de las entrevistas y los relatos de las personas jóvenes de sectores populares, identifiqué tres narrativas del yo presentes de manera combinada en torno a las cuales se configuran y articulan sus agencias y sus experiencias en instituciones, las cuales propician el ejercicio de derechos en el AMBA:

a) Narrativas de descubrimiento: el encuentro y la decisión de sostener un vínculo con estas instituciones se deben comprender en relación a sus procesos de: las vulnerabilidades -en sus dimensiones individuales, intersubjetivas e institucionales-; las debilidades en el acceso a soportes afectivos, simbólicos y materiales; los sentimientos de inseguridad, profundizados por su impacto en las redes de sociabilidad barrial; las ausencias, faltas de respeto, abusos de poder, injusticias y estigmatizaciones protagonizados por agentes de otras instituciones estatales con las que se relacionan durante sus vidas

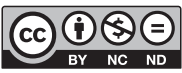

Rev. Reflexiones 98 (2) Julio-Diciembre, 2019: 141-158, E-ISSN: 1659-2859 
(algunas escuelas y agentes de fuerzas de seguridad). Frente a estas vulnerabilidades, los encuentros con instituciones, organizaciones y referentes sociales que habilitan distintos tipos de soportes afectivos, materiales y simbólicos, son significados como verdaderos descubrimientos. Estos escenarios se constituyen como refugios subjetivos en donde encuentran espacios de diálogo, se sienten personas reconocidas y se apropian de repertorios de acción con los cuales pueden (re)construir nuevas formas de convivencia con otras personas y de autonomía individual.

b) Narrativas de reorientación moral: a partir de relaciones de confianza, compromisos e identificaciones en estas instituciones, las personas jóvenes (re)construyen sus seguridades ontológicas, sus autoconfianzas y sus agencias. En torno a diversos acontecimientos biográficos vinculados a sus experiencias institucionales, redefinen narrativamente los marcos referenciales que orientan al yo, aquello que para ellas y ellos vale la pena hacer: la vida buena.

c) Narrativas de transformación colectiva: en estos espacios públicos, las y los jóvenes construyen sus identidades personales a partir del diálogo entre sus perspectivas internas y externas, subjetivas e intersubjetivas. De esta manera, gracias a la apertura de su ser ético, estético y político, pueden conformarse como individuos, reconocerse como seres genéricos y establecer una relación creativa con sí mismos, los otros y con su sociedad. Emergen así concepciones del poder y la agencia basadas en el vivir y actuar en común, como potencialidad de crear un colectivo, una comunidad, con la que pueden transformar sus realidades, recreando nuevas formas de igualdad. Aquí adquiere centralidad como marco referencial la parresía, que sintetiza el cuidado de sí y de otras personas, la moral y la ética, a partir de la escucha, la ayuda y el acompañamiento a otras y otros con experiencias o problemáticas similares a las vividas por ellas y ellos.

En estos escenarios sociales, institucionales, vinculares y simbólicos, las personas jóvenes conforman sus experiencias y sus procesos de individuación, (re)orientan y (re)construyen sus identidades, articulando diversas lógicas de la acción, soportes y marcos referenciales. Sus agencias se despliegan en una permanente y elástica tensión con múltiples fuentes de heteronomía -personas, grupos, instituciones, normas, valores- que contienen, en el doble sentido de limitar y sostener, sus identidades personales; y suministran soportes, los compromisos e identificaciones en relación a los cuales pueden (re)orientar sus marcos referenciales, prácticas y proyectos. Simultáneamente, en estas instituciones construyen diversas formas de autonomía, de subjetivación, de transformación individual y colectiva, que no se surgen de un yo sustancial y autocentrado, sino de vínculos y acuerdos intersubjetivos.

De esta manera, estas instituciones insertas en territorios socialmente vulnerabilizados, suministran a las y los jóvenes diversos soportes - simbólicos, materiales y, sobre todo afectivos- que les abren oportunidades para resignificar y ejercen sus derechos a la educación, la recreación, el trabajo regulado, el cuidado de sí y de los otros. Construyen así formas de ejercicio de la ciudadanía que no responden a definiciones teóricas o legales abstractas, sino que se anclan en sus experiencias, maneras cotidianas de convivir y proyectar con otras personas. Considero que la visibilización y la reflexión en torno a las experiencias y agencias juveniles en estas instituciones pueden contribuir a recrear y fortalecer las políticas sociales que trabajan con jóvenes de sectores populares, dirigidas a disminuir las desigualdades y las vulnerabilidades presentes en sus biografías y en sus barrios.

Me gustaría culminar el artículo con una reflexión -situada en el plano teórico-político de las ciencias sociales- surgida de esta experiencia de investigación. La consideración por las tensiones entre heteronomías y autonomías, constitutivas del yo y las agencias de las personas jóvenes, no implica renunciar a la crítica rigurosa ni a proyectos políticos dirigidos a desnaturalizar y transformar las condiciones; así como las prácticas de dominación o injusticia presentes muchas veces en las estructuras sociales y las instituciones en las que viven. Por el contrario, coincidiendo con Mahmood (2006),

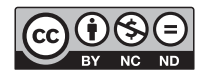

Rev. Reflexiones 98 (2) Julio-Diciembre, 2019: 141-158, E-ISSN: 1659-2859 
esta perspectiva permite ampliar una concepción normativa y limitada de la crítica -entendida meramente como la deconstrucción efectiva de la posición del oponente, exponiendo las inconsistencias o los límites de su lógica- hacia un sentido más potente, que parte de la reflexión y la transformación de nuestros propios prejuicios, identidades y prácticas. El encuentro con las visiones del mundo de otros yos crea así nuevas oportunidades para identificar, comprender e incorporar en nuestros análisis y acciones políticas otras formas de agencia, de autonomía, de transformación individual y colectiva que -desde las anteojeras de nuestras certezas teóricas e ideológicas- muchas veces nos resultan invisibles o inocuas.

\section{Notas}

1. Se denomina AMBA al área que comprende a la Ciudad Autónoma de Buenos Aires y parte de la Provincia de Buenos Aires. Produce el 48\% del producto bruto argentino, allí viven 15 millones de personas, aproximadamente un tercio de la población nacional. Sin embargo, es la segunda región más pobre de Argentina (la primera es el noroeste), con la mayor heterogeneidad distributiva del país (Piovani y Salvia 2018).

2. Consideramos que resulta inadecuado homogeneizar a los sectores populares mediante categorías como "pobreza" o "marginalidad", que muchas veces contribuyen a procesos de estigmatización, sobredimensionando sus carencias e invisibilizando sus agencias individuales y colectivas (Semán y Ferraudi Couto 2016).

3. Proyecto UBACyT 2016 Mod II 20020150200080BA. Director: Pablo Francisco Di Leo; codirectora: Ana Josefina Arias. Financiado por la Universidad de Buenos Aires (UBA). Sede: Instituto de Investigaciones Gino Germani, Facultad de Ciencias Sociales, UBA.

4. Si bien en el estudio también se entrevistaron a referentes de cada una de las instituciones, en este artículo presento resultados del análisis de las entrevistas y relatos de las personas jóvenes, ya que las preguntas-problema de investigación trabajadas aquí se centran en sus experiencias y agencias.

5. Durante todo el desarrollo de la investigación se siguieron los resguardos éticos fijados por la Resolución N $N^{\text {2 } 2857 / 2006 ~ d e l ~ C o n s e j o ~ N a c i o n a l ~ d e ~ I n v e s t i g a c i o n e s ~ C i e n t i ́ f i c a s ~ y ~ T e ́ c n i c a s ~}$ (CONICET), Argentina, para preservar el anonimato, la identidad y la integridad moral, social, psicológica y cultural de los sujetos que participaron en las entrevistas de manera informada y voluntaria, asegurando la confidencialidad de sus respuestas. Al requerirles su colaboración, se leyeron y entregaron a las personas jóvenes y a las autoridades y referentes de las instituciones los correspondientes Consentimientos Informados, previamente aprobados por un Comité de Ética. En la exposición de los resultados se toman los resguardos necesarios para preservar las identidades individuales e institucionales, reemplazando todos los nombres propios por pseudónimos.

6. Ver datos de las personas jóvenes entrevistadas en la Tabla 1.

7. Las villas son urbanizaciones informales que resultan de ocupaciones no planificadas de tierra urbana vacante. Se caracterizan por una alta densidad poblacional y por la presencia de viviendas construidas con materiales precarios y producto de desechos. Sus habitantes suelen ser trabajadores informales o con baja calificación a quienes se les suele atribuir características estigmatizantes (Cravino 2008).

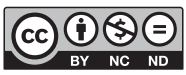


8. A agência, deste ponto de vista, é entendida como a capacidade de cada pessoa para realizar os seus interesses individuais, em oposição ao peso do costume, tradição, vontade transcendental ou outros obstáculos individuais e coletivos. Portanto, o objetivo humanista da autonomia e expressão das capacidades individuais constitui o substrato, as cinzas dormentes que poderão desatar em chamas sob a forma de um ato de resistência quando as condições assim o permitam (Mahmood 2006, 127).

\section{Referencias}

Araujo, Kathya y Danilo Martuccelli. 2012. Desafíos comunes. Retrato de la sociedad chilena y sus individuos. Santiago de Chile, Chile: LOM Ediciones.

Arendt, Hannah. 1993. La condición humana. Buenos Aires, Argentina: Editorial Paidós.

Auyero, Javier y María Fernanda Berti. 2013. La violencia en los márgenes. Una maestra y un sociólogo en el conurbano bonaerense. Buenos Aires, Argentina: Editorial Katz.

Butler, Judith. 2012. Dar cuenta de sí mismo: violencia ética y responsabilidad. Buenos Aires, Argentina: Amorrortu Editores.

Castoriadis, Cornelius. 1997. El mundo fragmentado. La Plata, Argentina: Editorial Altamira.

Chaves, Mariana, Sebastián G. Fuentes y Luisa Vecino. 2016. Experiencias juveniles de desigualdad. Fronteras y merecimientos en sectores populares, medios altos y altos. Buenos Aires, Argentina: Grupo Editor Universitario.

Cravino, María Cristina. 2008. Vivir en la villa: relatos, trayectorias y estrategias habitacionales. Los Polvorines, Argentina: Universidad Nacional de General Sarmiento.

Di Leo, Pablo Francisco y Ana Clara Camarotti. 2017. «Relatos biográficos y procesos de individuación juveniles en barrios marginalizados de Argentina». Revista Latinoamericana de Ciencias Sociales, Niñez y Juventud 15 (2): 1021-1034. DOI:10.11600/1692715x.1521530082016

Di Napoli, Pablo. 2018. «Sentidos y usos del "mirar mal" como eje de conflicto entre estudiantes». Revista Reflexiones 97 (2): 71-85. https://doi.org/10.15517/rr.v97i2.32281

Dubet, François. 2013. El trabajo de las sociedades. Buenos Aires, Argentina: Amorrortu Editores.

- 2017. Lo que nos une. Cómo vivir juntos a partir de un reconocimiento positivo de la diferencia. Buenos Aires, Argentina: Siglo XXI Editores.

Ema López, José Enrique. 2004. «Del sujeto a la agencia (a través de lo político)». Athenea Digital, 5 (primavera): 1-24. http://atheneadigital.net/article/view/n5-ema/114-pdf-es

Foucault, Michel. 1996. Hermenéutica del sujeto. La Plata, Argentina: Editorial Altamira.

Gentile, María Florencia. 2017. Biografías callejeras: cursos de vida de jóvenes en condiciones de desigualdad. Buenos Aires, Argentina: Grupo Editor Universitario.

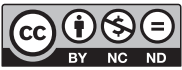

Rev. Reflexiones 98 (2) Julio-Diciembre, 2019: 141-158, E-ISSN: 1659-2859 
Giarracca, Norma y Karina Bidaseca. 2004. «Ensamblando las voces: los actores en el texto sociológico». En Metodologías cualitativas en ciencias sociales. Modelos y procedimientos de análisis, coordinado por Ana Lía Kornblit, 35-46. Buenos Aires, Argentina: Editorial Biblos.

Giddens, Anthony. 1991. Modernity and self-identity: Self and identity in the late modern age. Cambridge, UK: Polity Books.

Heller, Agnes. 2017. «Reflections on Ethics and the dynamics of personal identity». En Vulnerabilidades e Resistências na Integralidade do Cuidado: pluralidades multicêntricas de ações, pensamentos e a (re) forma do conhecimento, organizado por Roseni Pinheiro, Tatiana Engel Gerhardt y Felipe Dutra Asensi, 17-26. Rio de Janeiro, Brasil: CEPESC, IMS/UERJ, ABRASCO.

Kessler Gabriel y Matías Bruno. 2018. «Inseguridad y vulnerabilidad al delito». En La Argentina en el siglo XXI. Cómo somos, vivimos y convivimos en una sociedad desigual, coordinado por Juan Ignacio Piovani y Agustín Salvia, 329-355. Buenos Aires Polity: Siglo XXI Editores.

Leclerc-Olive, Michèle. 2009. «Temporalidades de la experiencia: las biografías y sus acontecimientos». Iberofórum. Revista de Ciencias Sociales de la Universidad Iberoamericana IV (8): 1-39. http:// www.redalyc.org/html/2110/211014822001/

Mahmood, Saba. 2006. «Teoria feminista, agência e sujeito liberatório: algumas reflexões sobre o revivalismo islâmico no Egipto». Etnográfica 10 (1): 121-158. http://www.scielo.mec.pt/scielo. php?script=sci_arttext\&pid=S0873-65612006000100007

Martuccelli, Danilo. 2007. Cambio de rumbo. La sociedad a escala del individuo. Santiago de Chile, Chile: LOM Ediciones.

Meccia, Ernesto. 2016. El tiempo no para. Los últimos homosexuales cuentan la historia. Buenos Aires, Argentina: Editorial Universitaria de Buenos Aires.

Núñez, Pedro y Lucía Litichever. 2015. Radiografías de la experiencia escolar. Buenos Aires, Argentina: Grupo Editor Universitario.

Paulín, Horacio Luis y Marina Tomasini, coord. 2014. Jóvenes y escuela. Relatos sobre una relación compleja. Córdoba, Argentina: Editorial Brujas.

Piovani, Juan Ignacio y Agustín Salvia, ed. 2018. La Argentina en el siglo XXI. Cómo somos, vivimos y convivimos en una sociedad desigual. Buenos Aires, Argentina: Siglo XXI.

Pujadas Muñoz, Juan José. 1992. El Método biográfico: el uso de las historias de vida en ciencias sociales. Madrid: Centro de Investigaciones Sociológicas.

Ricoeur, Paul. 1996. Sí mismo como otro. México D.F., México: Siglo XXI Editores.

Said, Shirly. 2018. «Jóvenes en Bachilleratos Populares: entre la individuación y la subjetivación política». Universitas, 28 (marzo-agosto): 141-157. http://dx.doi.org/10.17163/uni.n28.2018.07

Semán, Pablo y Cecilia Ferraudi Couto. 2016. «Los sectores populares». En La sociedad argentina hoy. Radiografía de una nueva estructura, compilado por Gabriel Kessler, 141-162. Buenos Aires, Argentina: Editorial Siglo XXI Editores.

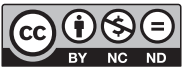


Strauss, Anselm L., Juliet Corbin y Eva Zimmerman. 2002. Bases de la investigación cualitativa: técnicas y procedimientos para desarrollar la teoría fundamentada. Medellín, Colombia: Universidad de Antioquia.

Taylor, Charles. 2006. Fuentes del yo. La construcción de la identidad moderna. Barcelona, España: Editorial Paidós Ibérica.

Vasilachis de Gialdino, Irene, coord. 2007. Estrategias de investigación cualitativa. Buenos Aires, Argentina: Editorial Gedisa.

Vommaro, Pablo. 2015. Juventudes y políticas en la Argentina y en América Latina: tendencias, conflictos y desafíos. Buenos Aires, Argentina: Grupo Editor Universitario. 